\section{OC-094 SYMPTOM TRIGGERED PHARMACOTHERAPY FOR ACUTE UNPLANNED ALCOHOL WITHDRAWAL CAN BE BOTH CLINICALLY AND COST-EFFECTIVE IN A HOSPITAL SETTING: EXPERIENCE FROM A SPECIALIST HEPATOLOGY UNIT}

\author{
doi:10.1136/gut.2011.239301.94
}

L Glover, * P Collins, F Gordon, H Karen, V Hunt, J Portal, S Saleem, C A McCune Department of Hepatology, University Hospitals Bristol NHS Foundation Trust, Bristol, UK

Introduction In recent guidance, NICE (2010) recommend a symptom-triggered (STW) pharmacological dosing regimen to treat AAW as both clinically and cost effective. ${ }^{1}$ Supporting evidence is from a small number of studies largely undertaken in specialised addiction units and a paucity of published evidence from other settings remains. With this in mind our liver unit formulated and piloted a STW regimen using chlordiazepoxide (CDP). Our aim was to evaluate STW and explore potential cost savings.

Methods The STW regimen allowed a maximum daily dose of $240 \mathrm{mg}$ CDP without specialist review. Pharmacotherapy was tailored to the patient's requirements as determined by the severity of their withdrawal signs and symptoms using a designated questionnaire (modified CIWA-AD2). Patients were scored hourly or 4 hourly according to protocol score. Data was collected prospectively over a 10-month period (Nov 09 Aug 10) and case note review undertaken in those previously admitted with AAW (all managed on a fixed dose regimen).

Results 135 individuals had STW, each consuming a mean of 28 units of alcohol/day. Most ( $\mathrm{n}=78,58 \%)$ were admitted with uncomplicated AAW, although over a third had significant liver disease ( $n=51,38 \%) .6(4 \%)$ had no significant liver disease but other serious co-morbidity requiring admission. 41 (30.4\%) were previously admitted with AAW. The mean total dose of CDP prescribed during STW was $70.6 \mathrm{mg}$ c.f. $541.6 \mathrm{mg}$ on a fixed dose regimen. A significant number ( $n=53,39 \%)$ of STW patients did not require CDP at all, allowing early discharge. Patients on STW required CPD on average for shorter duration (3.11 days vs 7.52 days for fixed dose regimen). In the group admitted with uncomplicated AAW, we estimate within one ward cost savings of at least $£ 220 \mathrm{k}$ per annum in bed days alone. Nursing staff welcomed the STW protocol, citing improved patient care and a reduction in violence and aggressive behaviour on the ward.

Conclusion Our results support the recommendations made by NICE (2010) that symptom-triggered regimens are associated with lower doses of benzodiazepines, shorter treatment duration and cost-savings, especially in those with uncomplicated AAW. Designated questionnaires (such as CIWR-AD) are a useful adjunct to clinical experience.

Competing interests None.

Keywords acute alcohol withdrawal, benzodiazepine, chlordiazepoxide, CIWA-AD, fixed dose, liver disease, symptom triggered.

\section{REFERENCES}

1. National Institute for Health and Clinical Excellence. Alcohol Use Disorders: Diagnosis and Clinical Management of Alcohol-Related Physical Complications. CG100. London: National Institute for Health and Clinical Excellence 2010.

2. Foy A, McKay S, Ling $S$, et al. Clinical use of a shortened alcohol withdrawal scale in a general hospital. Intern Med J 2006;36:150-4. 